\title{
Dependence of quantum-dot formation on substrate orientation studied by magnetophotoluminescence
}

\author{
J. Maes, ${ }^{\text {a) }}$ M. Hayne, and V. V. Moshchalkov \\ Laboratorium voor Vaste-Stoffysica en Magnetisme, Katholieke Universiteit Leuven, Celestijnenlaan 200D, \\ B-3001 Leuven, Belgium
}

\begin{abstract}
A. Patanè, M. Henini, L. Eaves, and P. C. Main
School of Physics and Astronomy, University of Nottingham, Nottingham, NG7 2RD United Kingdom
\end{abstract}

(Received 8 May 2002; accepted for publication 25 June 2002)

\begin{abstract}
We have investigated the substrate orientation-dependence of InAs/GaAs quantum dot growth by photoluminescence spectroscopy in very high magnetic fields. An abrupt change from one-dimensional to three-dimensional charge confinement is observed for InAs deposited on (100) GaAs. On the tilted (311)B substrates, the quantum dot morphology is different, resulting in a weaker charge confinement that gradually increases with the amount of deposited InAs. At 1.9 monolayers, the quantum-dot confinement on this substrate orientation is as effective as for the (100) oriented substrates. By studying the confinement of the charges in samples with quantum dots at different stages of development, we are able to give insight into the quantum-dot formation process. (C) 2002 American Institute of Physics. [DOI: 10.1063/1.1501160]
\end{abstract}

The formation of self-assembled quantum dots on (100) substrates under certain growth conditions is intensively investigated. ${ }^{1}$ Less well studied are heterostructures grown on a tilted substrate. In the $\mathrm{In}_{0.5} \mathrm{Ga}_{0.5} \mathrm{As} / \mathrm{GaAs}$ system for example, corrugated quantum well structures can be formed if a (311)A GaAs substrate is used, ${ }^{2}$ whereas for similar growth conditions on the (311)B substrate quantum dots are observed. ${ }^{3}$ In this study we examine the highly strained InAs/GaAs islands on flat (100) and tilted, i.e., (311)B, substrates by photoluminescence (PL) spectroscopy in high magnetic fields. This technique has proven to be a useful tool in the investigation of charge confinement. ${ }^{4}$ The PL energy shift in a magnetic field is a measure of the confinement of the charge carriers in the plane perpendicular to the field direction. For a strongly localized charge carrier magnetic field induced confinement will only be reached at very high fields, reducing the influence of the applied field on the energy levels, and resulting in a small diamagnetic shift. For charges that are weakly localized in the plane perpendicular to the applied field, a magnetic field will have a much larger influence, giving a large diamagnetic shift. With this method, we can, for example, distinguish between wetting layer (WL) and quantum-dot confinement by applying a field perpendicular to the plane of the sample (Fig. 1, inset). We have applied this methodology to the confinement of monolayers of InAs on (100) and (311)B oriented substrates. We find that for the (100) substrates there is a two-dimensional (2D) to three-dimensional (3D) transition, whereas on the (311)B substrates the dots gradually evolve from wetting layer fluctuations.

The InAs/GaAs heterostructures were grown by molecular beam epitaxy on (100) and (311)B oriented GaAs substrates. ${ }^{5,6}$ After a $0.5 \mu \mathrm{m}$ GaAs buffer layer, an amount of InAs was deposited that ranges from 1.4 to 1.9 monolayers

${ }^{a)}$ Electronic mail: jochen.maes@fys.kuleuven.ac.be
(ML) in steps of $0.1 \mathrm{ML}$. The growth temperature was $480^{\circ} \mathrm{C}$. A $25 \mathrm{~nm} \mathrm{GaAs}$ capping layer completes the structures. PL measurements were performed at $4.2 \mathrm{~K}$ in pulsed magnetic fields up to $50 \mathrm{~T}$. During a $25 \mathrm{~ms}$ magnetic field pulse, up to five spectra can be taken. At the peak of the pulse, where the field variation in time is small, a typical field resolution of $\pm 0.3 \%$ is obtained, while during the up sweep and down sweep the field resolution is of the order of $\pm 3 \%$. The photon integration time ranges from 0.5 to 1.0 ms. The magnetic field, $B$, can be applied parallel (\|) or perpendicular $(\perp)$ to the sample growth direction $(z)$. Excitation of the samples is achieved by a Nd:yttritiumaluminum-garnet laser operating at $532 \mathrm{~nm}$ via a $200 \mu \mathrm{m}$ core optical fiber with a power density of $1-10 \mathrm{Wcm}^{-2}$. The luminescence, collected by six surrounding fibers, is ana-

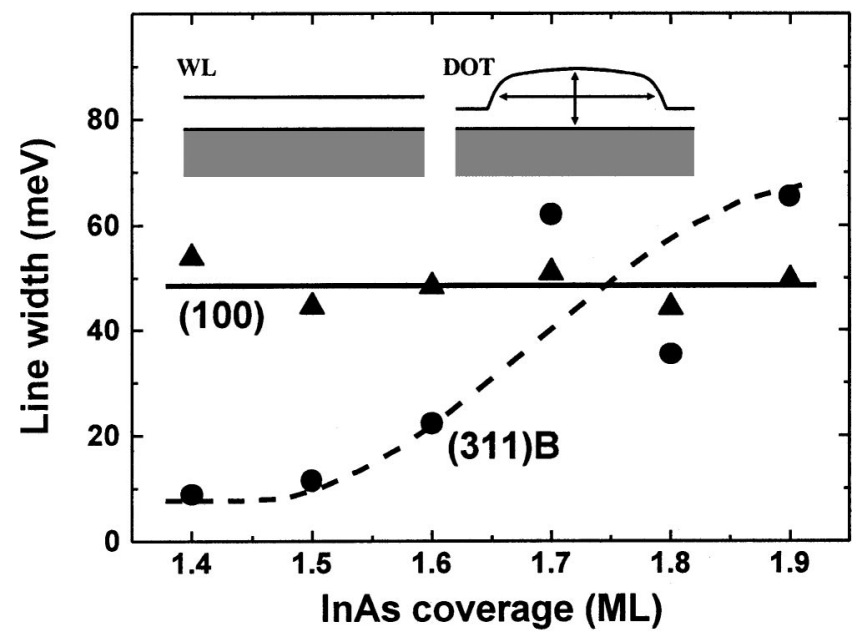

FIG. 1. Linewidth as a function of the InAs coverage for (100) - triangles and $(311) \mathrm{B}$ - circles oriented substrates. The lines are a guide to the eye. The schematic inset shows that a quantum dot has a stronger in-plane confinement than a quantum well but has less confinement in the growth direction. 


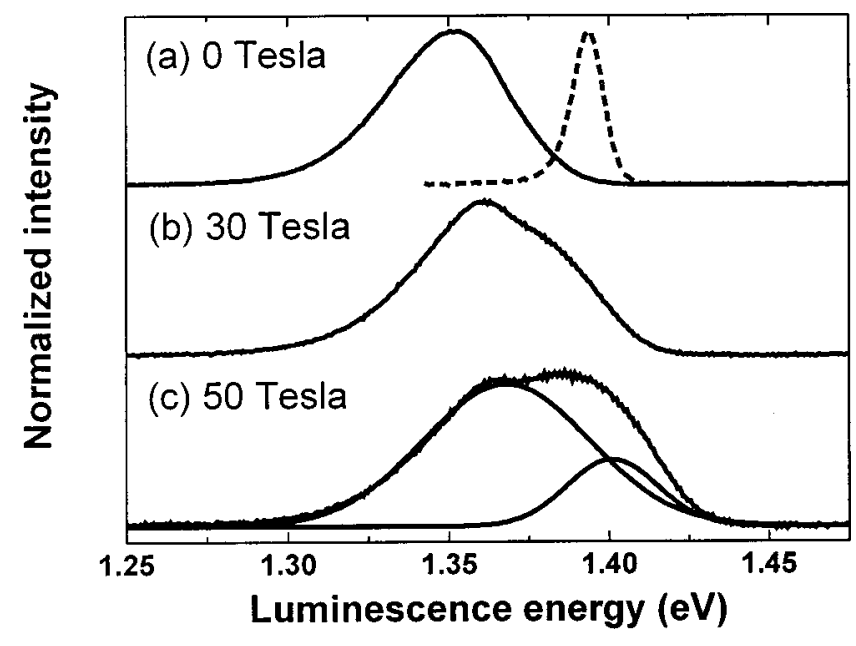

FIG. 2. Photoluminescence spectra of 1.5 ML of InAs deposited on (100) GaAs (a) without field and with a magnetic field of (b) 30 and (c) $50 \mathrm{~T}$ applied parallel to the growth direction. The $50 \mathrm{~T}$ data clearly reveal that the luminescence is comprised of two peaks, a broad $(51 \mathrm{meV})$ low-energy peak attributed to the dots and a narrow $(28 \mathrm{meV})$ high-energy peak attributed to the wetting layer. The dashed line in (a) is the zero-field PL spectrum of 1.5 ML of InAs on the (311)B substrate.

lyzed in a $0.25 \mathrm{~m}$ spectrometer with a liquid-nitrogen cooled InGaAs detector.

The dependence of the zero-field PL-line positions on the coverage can be found in Ref. 5. The substrate orientation has no significant influence on the shift of the peak, which shows a strong redshift with increasing coverage. We attribute this to a reduction of the band gap by strain relaxation, rather than a reduction in vertical confinement (Fig. 1, inset). We will show that the latter has a very moderate effect on the confinement as determined by the diamagnetic shift, and behaves differently for the two substrate orientations.

We first concentrate on the systems grown on the (100) oriented substrates. Here, the broadness of the PL reveals the presence of quantum dots at a coverage of as little as $1.4 \mathrm{ML}$ (Fig. 1). For 1.5 ML, a barely asymmetric PL line is observed at zero field as shown in Fig. 2(a). By $30 \mathrm{~T}$ a clear shoulder is visible, but it is only at the highest magnetic field that the two contributions to the luminescence can be clearly distinguished. There is a broad peak, which is characteristic of dot luminescence, and a narrow one that we ascribe to the wetting layer. This distinction can only be made at high fields because the PL energy of the wetting layer has a larger shift in magnetic fields due to the lack of lateral spatial confinement (Fig. 1, inset). The presence of the asymmetric PL underlines the important role of the wetting layer and indicates that dot formation in this low-coverage regime is still nonuniform, leaving parts of the sample without developed dots. This explains the late appearance (at 1.7 ML) of the streaky-to-spotty transition in the reflection high-energy electron diffraction pattern ${ }^{6}$ that indicates the transition from $2 \mathrm{D}$ to $3 \mathrm{D}$ growth. Further evidence that recombination in the wetting layer is persistent for low coverages can be found in Fig. 3(a) where the diamagnetic energy shift, $\Delta E$, between 0 and $50 \mathrm{~T}$ is plotted against the InAs coverage for $B \|_{z}$ and $B \perp z$. At low coverages, the diamagnetic shift with $B \| z$ is large indicating that a considerable contribution to the luminescence comes from weakly confined charges, as in a quantum well. The crossover to a symmetric PL line and the

Downloaded 19 Aug 2002 to 137.108.143.7. Redistribution subje

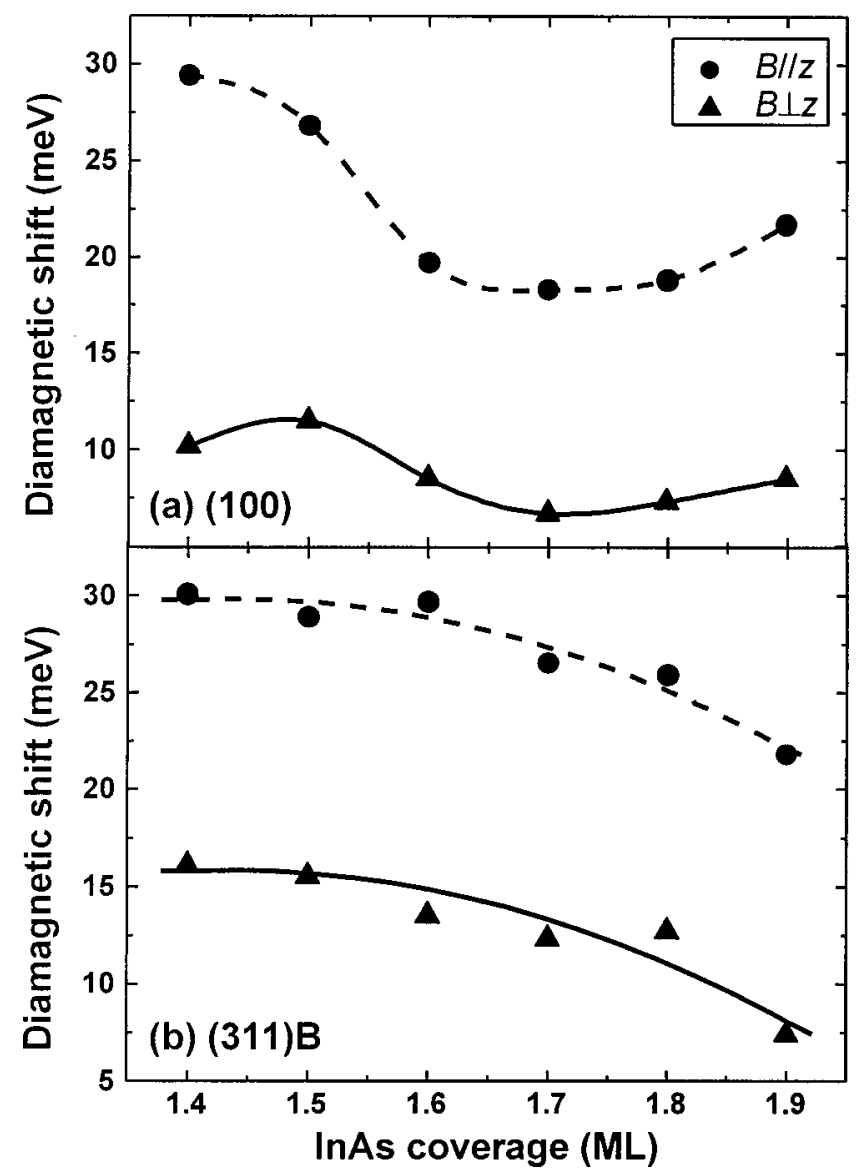

FIG. 3. The diamagnetic energy shift, $\Delta E$, between 0 and $50 \mathrm{~T}$ for both magnetic field directions as a function of the amount of InAs deposited on (a) (100) and (b) (311)B oriented GaAs substrates. For (100) GaAs, the sudden drop in $\Delta E$ for $B \| z$ at low coverage and the increase at high coverage are eye catching. For InAs on (311)B GaAs, $\Delta E$ gradually decreases with coverage.

sudden drop in diamagnetic shift for $B \|_{z}$ at $1.6 \mathrm{ML}$ indicate that the luminescence is dominated by the dots from 1.6 ML on. In this regime, the charges are well confined inside the quantum dots, and as the amount of InAs coverage increases, the decrease in localization due to the larger dot size leads to a gradual increase in diamagnetic shift [Fig. 3(a)]. For $B \perp z$ the changes in diamagnetic shift are considerably weaker but a similar trend is observed. With the field in this direction the diamagnetic shift is influenced by both the spatial confinement in the growth direction and the confinement in the plane, though the stronger of the two, typically the confinement in the growth direction, should dominate. We note that according to our earlier-mentioned simple model the $z$ confinement should be reduced when the dots form (Fig. 1, inset). The data in Fig. 3(a) show that the effect of the increased lateral confinement is strongly but not completely damped by the reduced $z$ confinement.

On the (311)B substrate the natural surface modulation limits the in-plane atom diffusion and thus hinders quantumdot formation. ${ }^{5,7}$ At low coverage, one then expects that the observed luminescence can be ascribed to the wetting layer. Indeed, the line is narrow but is asymmetric [Fig. 2(a)]. Furthermore, the PL line shape remains unchanged for all fields, indicating that in contrast to the (100) samples with 1.4 and 1.5 ML, a contribution from developed quantum dots is negligible. As the amount of InAs deposited increases, the PL to AlP license or copyright, see http://ojps.aip.org/aplo/aplcr.jsp 
line broadens (Fig. 1) but remains asymmetric up to $1.8 \mathrm{ML}$, thus revealing the persistence of the wetting layer at high coverages. The gradual decrease in diamagnetic shift [Fig. $3(b)]$ demonstrates that the charges become more and more confined in all spatial directions and that there is an evolution from wetting layer to dot behavior. The fact that there is also no increase in $\Delta E$ even for high coverage, leads us to believe that the charges are not yet fully confined in the dots up to $1.8 \mathrm{ML}$. Due to the absence of long-range ordering on the (311)B high-index substrates, ${ }^{7}$ any layer that is grown on it will tend to develop fluctuations in its thickness. In addition, the step edges on the (311)B GaAs cause a certain amount of strain relaxation in the InAs, resulting in weaker strain fields. ${ }^{3}$ These two features cause the growth mode to be different from the Stranski-Krastanov mode in which a sharp transition from 2D to 3D growth occurs. Instead, we believe the wetting layer surface fluctuations gradually evolve into quantum dots. These surface fluctuations in the InAs wetting layer attract the charges, but the attractive potential is much weaker than that of developed quantum dots causing a spillover of the electron and hole wave functions into the rest of the wetting layer. In this case there is no discernible difference in the change in $\Delta E$ for $B \| z$ and $B \perp z$, indicating that there is no significant change in confinement in the $z$ direction, and that the dot formation in (100) and (311)B directions is rather different.

The sample with 1.9 ML of InAs is the first of the (311)B set that has a symmetric line shape. Its linewidth is broad and comparable to, though slightly larger than, that of the (100) samples which have fully developed dots, and the diamagnetic shift for both field directions is small. These are all indications that the InAs quantum dots are fully developed from 1.9 ML on if deposited on a GaAs (311)B substrate. Furthermore, the diamagnetic shifts are almost identical to that of the $1.9 \mathrm{ML}$ on (100) sample. This shows that the quantum dots grown on the different substrates have a similar size. This is consistent with Ref. 5, where the dot diameter $d$ and height $h$ for similar but uncapped systems were found to be $d=15$ and $18 \mathrm{~nm}$ and $h=1.5$ and $1.4 \mathrm{~nm}$ for 1.8 ML of InAs on (100) GaAs and (311)B GaAs, respectively. The small difference in linewidth for 1.9 ML on (100) compared to (311)B can be attributed to a larger dot size distribution due to the modulated (311)B substrate.

We have studied the formation of InAs QDs in GaAs with magnetophotoluminescence. On (100) substrates the recombination of charges in the wetting layer remains important up to 1.5 ML. On (311)B substrates the abrupt StranskiKrastanov 2D-3D transition that holds for flat surfaces is replaced by a slow evolution from wetting layer surface fluctuations to developed quantum dots, which is completed by 1.9 ML. It is expected that this understanding of the QD formation process will lead to improvements in the performance of QD-based devices.

This work is supported by the FWO-Vlaanderen, the Flemish GOA, and the Belgian IUAP programes, the VIS 00/001 project of the KU Leuven, and the NANOMAT project of the EC Growth Program Contract No. G5RD-CT2001-00545.

\footnotetext{
${ }^{1}$ For a review, see D. Bimberg, M. Grundmann and N. N. Ledentsov, Quantum Dot Heterostructures (Wiley, Chichester, 1999).

${ }^{2}$ M. Wassermeier, J. Sudijono, M. D. Johnson, K. T. Leung, B. G. Orr, L. Däweritz, and K. Ploog, Phys. Rev. B 51, 14721 (1995).

${ }^{3}$ P. O. Vaccaro, M. Hirai, K. Fujita, and T. Watanabe, J. Phys. D 29, 2221 (1996).

${ }^{4}$ M. Hayne, J. Maes, Y. M. Manz, O. G. Schmidt, K. Eberl, and V. V. Moshchalkov, Appl. Phys. Lett. 79, 45 (2001); M. Hayne, R. Provoost, M. K. Zundel, Y. M. Manz, K. Eberl, and V. V. Moshchalkov, Phys. Rev. B 62, 10324 (2000)

${ }^{5}$ A. Polimeni, A. Patanè, M. Henini, L. Eaves, P. C. Main, S. Sanguinetti, and M. Guzzi, J. Cryst. Growth 202, 276 (1999).

${ }^{6}$ S. Sanguinetti, G. Chiantoni, E. Grilli, M. Guzzi, M. Henini, A. Polimeni, A. Patanè, L. Eaves, and P. C. Main, Europhys. Lett. 47, 701 (1999).

${ }^{7}$ P. Moriarty, Y.-R. Ma, A. W. Dunn, P. H. Beton, M. Henini, C. McGinley, E. McLoughlin, A. A. Cafolla, G. Hughes, S. Downes, D. Teehan, and B. Murphy, Phys. Rev. B 55, 15397 (1997).
} 